\title{
Pica Eating Disorder
}

National Cancer Institute

\section{Source}

National Cancer Institute. Pica Eating Disorder. NCI Thesaurus. Code C92566.

An eating disorder characterized by the persistent eating of nonnutritive substances such as clay or soil; this behavior must be inappropriate to the level of the individual's development. 